\title{
Construção participativa de descritores para avaliação dos núcleos de prevenção de acidentes e violência, Brasil
}

\author{
Suely Ferreira Deslandes ${ }^{1}$ e Marcela Pinto Lemos ${ }^{1}$
}

Como citar Deslandes SF, Lemos MP. Construção participativa de descritores para avaliação dos núcleos de prevenção de acidentes e violência, Brasil. Rev Panam Salud Publica. 2008;(24)(6):441-8.

RESUMO Objetivo. Descrever o processo de construção participativa de descritores para a avaliação dos Núcleos de Prevenção de Acidentes e Violência instituídos pelo Ministério da Saúde brasileiro, cuja missão é o acompanhamento e a mobilização de políticas públicas voltadas para a redução da morbimortalidade por causas externas.

Método. A técnica de grupo nominal foi a estratégia central empregada. Participaram 13 representantes dos núcleos dos estados do Rio de Janeiro e Espírito Santo.

Resultados. Foram produzidos 11 descritores para avaliação e foram eleitos cinco como prioritários: construção do diagnóstico situacional, articulação intra e intersetorial, monitoramento dos dados de mortalidade de acidentes e violência no município, apoio político, financeiro e administrativo (autonomia) e formação de equipe de trabalho (composição, dedicação e capacitação técnica).

Conclusões. Os descritores escolhidos revelam tanto a trajetória de construção de identidade desses núcleos e a maturidade de atuação do setor saúde nessa área como também antigos obstáculos a serem superados para a realização de ações mais efetivas de enfrentamento da violência no País.

Palavras-chave Causas externas, violência/prevenção \& controle, Brasil.

Desde o final dos anos 1980, as causas externas de morte - o conjunto das variadas expressões da violência e dos acidentes - assumiram, no Brasil, destaque no quadro de morbimortalidade. A redução da violência, por sua magnitude e complexidade, tem sido um dos maiores desafios das polí-

Instituto Fernandes Figueira, Departamento de Ensino. Enviar correspondência para Suely Ferreira Deslandes no seguinte endereço: Avenida Rui Barbosa 716, segundo andar, Flamengo, CEP 22250020, Rio de Janeiro, RJ, Brasil. Fone: +55-21-2554. 1796; fax: +55-21-2552.8898; e-mail: desland@iff. fiocruz.br ticas públicas. Historicamente associadas ao âmbito da segurança pública, a violência e suas formas de expressão atingiram um patamar tal que passaram a demandar um esforço ampliado da sociedade para que sejam compreendidas e para que seja possível delinear ações capazes de enfrentar tal problema (1).

O setor de saúde, por seu papel estratégico no cuidado às vítimas, tem sido cobrado a incluir, em sua pauta organizacional, a prevenção das violências e dos acidentes, a promoção de ambientes seguros e de práticas de sociabilidade pautadas pela tolerância e pela diversidade, a oferta de atendimento multidisciplinar às vítimas $\mathrm{e}$ aos seus familiares e a articulação setorial e intra-setorial para a elaboração de ações visando à redução de tais eventos (2, 3). A década de 1990 foi marcada pela orientação, por parte da Organização Mundial da Saúde (OMS), de incorporar a violência e suas formas de prevenção nas agendas nacionais (4) a partir de ações realizadas pelas secretarias de saúde, bus- 
cando prover acolhimento, orientação e acompanhamento às vítimas através de parcerias intersetoriais com organizações não-governamentais (ONGs), conselhos tutelares, delegacias especializadas e centros de defesa dos direitos, entre outros (5-7).

Nessa trajetória, foi implantada, no Brasil, em 2001, a Política Nacional de Redução de Morbimortalidade por Acidentes e Violências (PNRMAV) (8). Apesar de uma implementação lenta e desigual nas diferentes regiões e municípios (9), a criação, em maio de 2004, de uma rede nacional de prevenção da violência e promoção da saúde (10), que instituiu vários núcleos de prevenção de acidentes e violências (NPAV), evidenciou o compromisso governamental com a implementação da PNRMAV. Os NPAV são constituídos por secretarias municipais e estaduais de saúde, instituições acadêmicas e ONGs, distribuídos em todas as regiões do País. O total de núcleos no primeiro semestre de 2007 era de 70 (11).

Os núcleos municipais são responsáveis por elaborar o plano municipal de prevenção da violência e promoção da saúde, promover e participar de políticas e ações intersetoriais e de redes sociais que busquem a prevenção da violência e a promoção da saúde, qualificar e articular a rede de atenção integral às pessoas vivendo em situações de violência e desenvolver ações de prevenção e promoção junto aos segmentos populacionais mais vulneráveis. Também devem garantir a implementação da notificação de maus-tratos e de outras violências, possibilitando a melhoria da qualidade da informação, e a participação de populações em situação de risco nas redes locais de atenção integral; estimular o desenvolvimento de estudos e pesquisas estratégicas; e capacitar os profissionais, movimentos e conselhos sociais para o trabalho de prevenção da violência em parceria com os pólos de educação permanente capazes de realizar a integração local e regional (10).

Os núcleos estaduais teriam atribuições similares, porém mais abrangentes, dado que seriam responsáveis pela articulação entre os municípios. Já os núcleos acadêmicos desenvolveriam indicadores e metodologias para o monitoramento e a avaliação das ações do Plano Nacional de Prevenção (a ser desenvolvido como estratégia de execução da PNRMAV), prestariam assessoria aos núcleos e apoiariam, elaborariam e realizariam eventos técnicos e publicações sobre a temática da violência, além de desenvolver tecnologias de comunicação entre os núcleos e apoiar o desenvolvimento de mecanismos de notificação de maustratos e outras violências (10). Passados 3 anos da criação desses núcleos, torna-se necessário analisar o processo de implantação e examinar a sua atuação e sustentabilidade políticoinstitucional, financeira e técnica.

Independentemente dos distintos propósitos que uma avaliação possa ter, os descritores e indicadores são ferramentas imprescindíveis para a análise do complexo percurso, quase nunca linear, entre o que foi proposto (objetivos, estratégias, resultados ou metas de um programa) e o que foi realizado ao término de determinado período $(12,13)$. Nessa avaliação, o papel dos descritores é apresentar os conteúdos, os pontos críticos, as características significativas para a manutenção e o funcionamento de um sistema (no caso a ação técnico-política dos NPAV), enquanto que os indicadores expressam a mensurabilidade (percentuais, taxas, etc.) (14). Numa concepção mais ampliada que a da mensurabilidade, os indicadores podem se referir aos aspectos tangíveis (aspectos observáveis) e intangíveis da realidade (elementos que só podem ser percebidos indiretamente, e geralmente através de estratégias qualitativas de pesquisa, como é o caso de consciência social, mudança de valores e atitudes) (14). Trata-se, enfim, de definir referentes empíricos que permitam medir ou inferir, de forma válida, por exemplo, o cumprimento das ações propostas, sua qualidade e efetividade $(15,16)$.

Os descritores apontam questões centrais que serão foco da avaliação; a partir deles, questões, variáveis e indicadores podem ser desenvolvidos ou selecionados. Se tomarmos, por exem- plo, a questão da qualidade de registro de determinada secretaria de saúde, poderíamos ter como descritor a "valorização das questões étnico-raciais na informação sobre eventos de violência" e como eventual indicador o "percentual de notificações de violência intrafamiliar em que a variável cor foi preenchida".

Os parâmetros para definir descritores e indicadores podem ser externos e até mesmo estranhos aos valores e idéias dos sujeitos envolvidos ou promotores das ações a serem avaliadas. Podem ser estipulados a priori, segundo um determinado "padrãoouro", considerado adequado para medir a excelência dos resultados e processos (16). As avaliações participativas criticam essas posturas e buscam minimizar a distância entre os avaliadores e os sujeitos beneficiários da avaliação, agregando a crítica externa à reflexão e à vivência interna do programa $(17,18)$.

A parceria entre os peritos em avaliação e os sujeitos responsáveis pela execução da ação, serviço ou programa que se avalia é, sob vários aspectos, uma opção estratégica $(14,19$, 20): com essa parceria, a avaliação ganha em pertinência, pois os sujeitos envolvidos têm a vivência da prática, permitindo elaborar perguntas "inteligentes" e exercer uma crítica das estratégias avaliativas. Além disso, a parceria desencadeia um processo de auto-reflexão por parte dos sujeitos sobre as ações e os rumos tomados e a tomar, e o processo propicia uma transferência de conhecimento e tecnologia de avaliação.

O presente artigo dedica-se, portanto, a descrever o processo participativo de elaboração e eleição de descritores para dar suporte à avaliação da atuação dos NPAV dos estados do Rio de Janeiro e Espírito Santo.

\section{MATERIAIS E MÉTODOS}

Neste trabalho, realizado em 2007, partiu-se do pressuposto de que os descritores construídos coletivamente e a partir de consenso poderão ganhar boa legitimidade e validade junto aos 
atores envolvidos nas ações dos NPAV. Espera-se que constituam a base para a elaboração de indicadores voltados à avaliação do processo de implantação e atuação desses núcleos e um ponto de partida para a avaliação dos demais núcleos brasileiros.

A técnica grupo nominal (TGN) foi a ferramenta metodológica central deste estudo. Trata-se de uma técnica quanti-qualitativa que permite sintetizar informações a fim de obter consensos entre especialistas sobre critérios, programas de formação e medidas de melhoria $(21,22)$. Nesta perspectiva, o conceito de especialista é ampliado, incluindo desde a expertise acadêmica até as pessoas cujas vivências sejam significativas para a questão em foco (23).

A TGN utiliza um roteiro padronizado de abordagem visando a comparar as diferentes propostas e estratificações. Geralmente envolve de cinco a nove participantes, cuja interação é controlada pelo mediador (24). A aplicação da técnica neste estudo consistiu em cinco passos básicos, conforme preconizado pela literatura $(21,24,25)$.

$\mathrm{Na}$ primeira etapa, solicitou-se aos participantes que enumerassem, por escrito, cinco descritores que julgassem essenciais à avaliação do processo de implantação e início da ação dos NPAV, tendo como horizonte o intervalo de 1 ano de atuação. Na segunda etapa, as respostas dos participantes foram transcritas num quadro. Na terceira etapa, cada participante defendeu seus argumentos, sem interrupções do grupo. Nesta fase algumas idéias foram agrupadas, e aquelas repetidas foram retiradas mediante negociação com os participantes, sendo então elaborada uma segunda versão da lista inicial.

A quarta etapa foi a de votação. Neste momento, cada participante escolheu os cinco itens que julgava mais importantes. Os cinco enunciados mais votados foram identificados, compondo uma terceira lista. $\mathrm{Na}$ quinta etapa, os participantes estabeleceram a prioridade dos cinco itens mais votados, do primeiro ao quinto. A estratificação final de prioridade foi estabelecida a partir da mediana dos votos. A fim de decidir, em caso de empate na ordenação, solicitou-se a cada membro que distribuísse 10 pontos entre os cinco itens, segundo sua ordem de prioridade (contanto que a soma total entre os pontos distribuídos dentre os cinco itens não ultrapassasse 10). A lista final revelou os cinco itens mais votados e a sua ordem de prioridade.

A sessão de aplicação da TGN envolveu representantes dos núcleos do Rio de Janeiro, Petrópolis e Niterói, todos no Estado do Rio de Janeiro, e de Cariacica, Vitória, Vila Velha e Serra, no Estado do Espírito Santo. Também participaram o Núcleo Estadual do Espírito Santo e o Núcleo Acadêmico do Centro Latino-Americano de Estudos de Violência e Saúde Jorge Careli (CLAVES), da Fundação Oswaldo Cruz. Cada um dos núcleos citados foi representado pelo seu coordenador. Aqueles representados por mais de um participante se organizaram em duplas ou grupos a fim de expressar apenas uma participação nas propostas apresentadas. Um dos nove gestores não pode participar até o final da dinâmica, envolvendo-se até a terceira etapa da técnica. A sessão contou com 13 participantes.

Após a tabulação dos dados, foi enviado relatório aos núcleos, descrevendo todas as etapas transcorridas e os resultados obtidos, almejando a correção de eventuais imprecisões e o ganho de validade do estudo.

Além dos procedimentos preconizados pela TGN, incorporamos a técnica de análise temática para a análise dos argumentos e explanações geradas pelos participantes do grupo para a produção do consenso. Esse procedimento se torna necessário quando há uma quantidade e riqueza de dados qualitativos que demandam aprofundamento. A associação da TGN à análise temática tem sido recorrente na literatura $(25,26)$.

Assim, a sessão da TGN foi gravada em áudio (com o consentimento dos participantes) e transcrita. O texto foi categorizado segundo cada argumento gerado no debate resgatandose descritivamente o processo de negociação discursiva que deu ori- gem a cada um dos quesitos considerados úteis à elaboração de descritores (27).

\section{RESULTADOS}

Na primeira rodada, os participantes produziram uma lista de 30 descritores (tabela 1). Na segunda etapa, alguns descritores mencionados foram reconhecidos pelos representantes dos NPAC como duplicados ou como contidos em outros mais abrangentes. Outros foram considerados inadequados à missão dos núcleos. A redação de alguns descritores também foi modificada pelo grupo com o objetivo de torná-los mais pertinentes ao momento em que se encontra a atuação dos NPAV (fase de implantação).

A redação do primeiro descritor foi imediatamente reconhecida pelo grupo como inadequada. Os participantes concluíram que a redução das taxas de mortalidade de acidentes e violência nos municípios não depende diretamente da ação dos NPAV. Contudo, desenvolver ações visando a garantir a qualidade da informação sobre tais dados estaria em seu escopo de atuação. Assim, a ação de "reduzir" as taxas foi substituída pela de "monitorar".

Posteriormente, o sexto descritor foi agregado ao primeiro, já que defendia a atualização do banco de acidentes e violências. O sexto descritor foi também considerado incompleto, pois a "disponibilidade de bancos de dados atualizados" abria a discussão acerca do papel do núcleo em relação aos bancos e acerca de quem deveria ter a responsabilidade de atualizar esses bancos. Havia núcleos cujos integrantes também compunham a equipe de vigilância de saúde do município, de forma que essas atribuições estavam justapostas. Noutras cidades, o NPAV interagia com o setor de vigilância, visando ao incremento da qualidade dos dados de causas externas. Assim, o grupo chegou ao consenso de que a expressão "monitoramento dos dados" resolvia o impasse, pois envolve a reconhecida função dos núcleos de acompanhamento das ações de vigilância de manter os dados atualiza- 
TABELA 1. Descritores elaborados para a avaliação dos Núcleos de Prevenção da Violência, Brasil, 2007

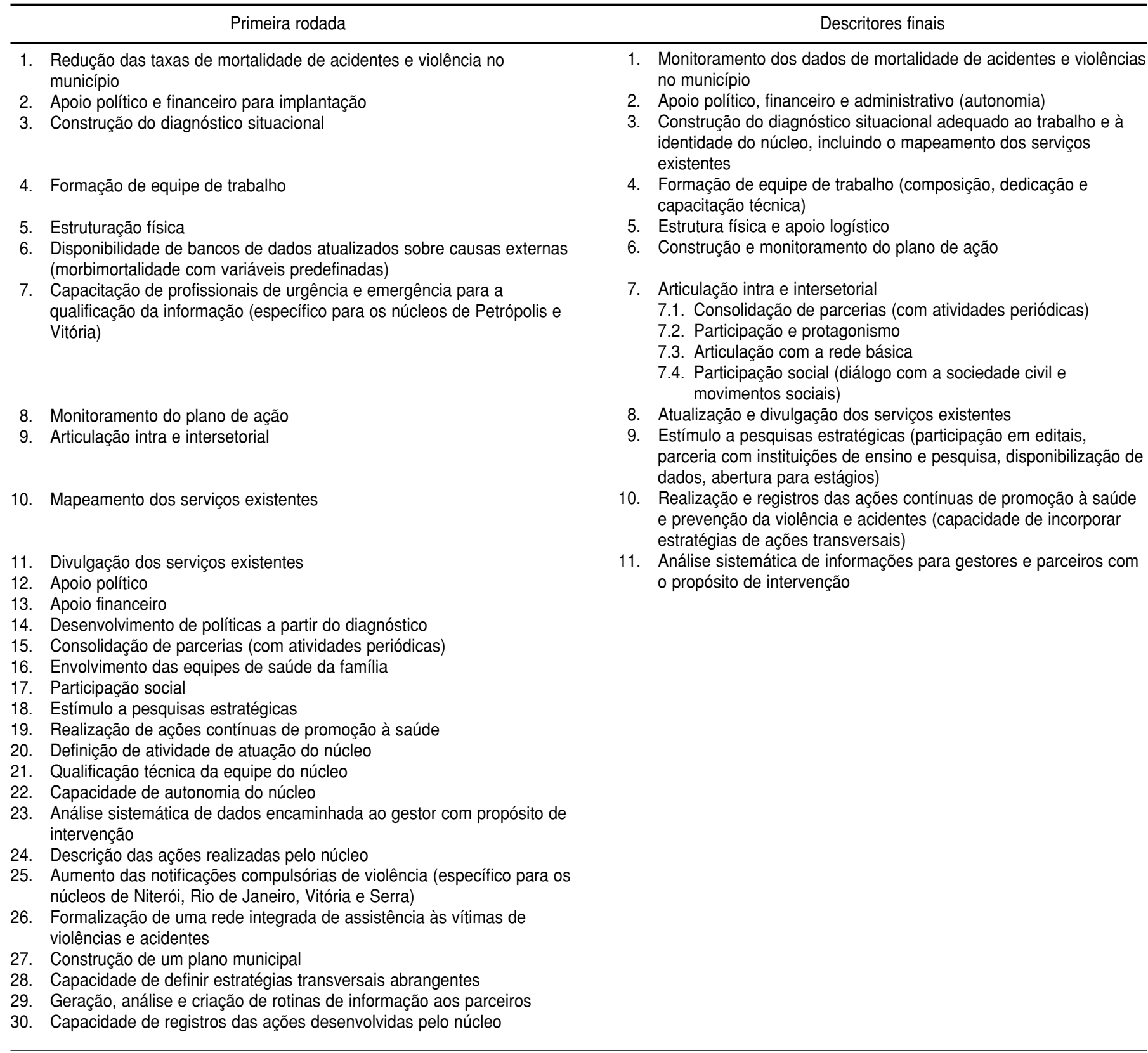

dos e inclui as diferentes competências de cada núcleo (descritor final 1, tabela 1).

Na seqüência do debate, considerou-se que os descritores 2,12 e 13 seriam agrupáveis. $\mathrm{O}$ grupo refletiu que os quesitos apoio político e financeiro são indissociáveis. Entretanto, alguns participantes lembraram que, embora conexos, esses itens possuem especifi- cidades, já que alguns NPAV têm o apoio financeiro do Ministério da Saúde, mas não têm o apoio político local, e, em outros casos, a gestão local (secretaria de saúde) pode estar efetivamente comprometida e o Ministério não ter repassado ainda a verba para o NPAV. Porém, a maioria concordou que é necessária a avaliação desse descritor de forma mais aprofundada, como duas necessidades que deveriam caminhar juntas para a avaliação das dificuldades encontradas pelos NPAV. Sendo assim, a sentença ainda incorporou o apoio administrativo, que leva em conta a capacidade de autonomia de tomada de decisões operacionais. Dessa forma, o descritor de número 22 também foi anexado na versão final (descritor final 2, tabela 1). 
O descritor "construção do diagnóstico situacional" foi considerado necessário, uma vez que constitui uma ferramenta fundamental para o começo da atuação de qualquer núcleo. O debate, entretanto, levantou argumentos sobre quais questões e variáveis deveriam ser incluídas em cada diagnóstico - se cada NPAV deveria adotar um mesmo modelo de diagnóstico ou se deveria haver autonomia para a construção de modelos adequados ao perfil e identidade de atuação de cada NPAV. Um dos núcleos, por exemplo, apontou que sua vertente de atuação será pela via da promoção da saúde; portanto, o seu diagnóstico deveria ser diferenciado daquele de outro núcleo cuja prioridade de trabalho será a de monitorar os eventos violentos. O grupo agregou também o descritor de número 10 , já que concluiu que o mapeamento dos serviços existentes é uma ação estratégica do diagnóstico visando a constituir parcerias para viabilizar as futuras ações do NPAV (descritor final 3, tabela 1).

Durante o debate sobre o descritor 4, "formação de equipe", foi ponderado que os itens composição (diversidade da formação profissional dos membros), dedicação (horas de trabalho de cada membro) e capacitação técnica não poderiam deixar de ser avaliados, já que são fundamentais para a formação de um grupo de trabalho estável e qualificado. Nesse sentido, o descritor 21 foi também incorporado (descritor final 4, tabela 1).

O descritor "estruturação física" reflete as condições do NPAV em termos de mobiliário, equipamentos e salas, por exemplo, e incorporou em sua sentença a expressão de "apoio logístico" (suprimentos, acesso à Internet, assinatura de jornais para monitoramento de eventos violentos, etc.). $\mathrm{O}$ grupo concluiu que ambos os fatores retratam as condições de trabalho do NPAV e devem ser analisados em conjunto (descritor final 5, tabela 1).

Os descritores 7 e 25, desde a sua enunciação, foram considerados pelo grupo como particulares a certos NPAV, refletindo suas prioridades de trabalho.
O descritor 8, "monitoramento do plano de ação", foi reconhecido como uma atribuição típica dos núcleos estaduais. Assim, foi adicionada a ele a expressão "construção" para refletir a ação dos núcleos municipais. Os participantes ponderaram que esse plano ainda não foi elaborado, devendo ser construído de acordo com a realidade de cada núcleo, possibilitando a autoavaliação, o monitoramento externo das atividades desenvolvidas e a construção da identidade de cada NPAV. Nesse sentido, os descritores 20 e 27 foram considerados como tendo sido aí contemplados (descritor final 6, tabela 1).

O descritor "articulação intra e intersetorial" (descritor 9 da primeira rodada e 7 da lista final) foi aprovado por todos os participantes, discutindo-se quais itens deveriam ser incorporados para a sua melhor qualificação. Assim, o indicador 15, "consolidação de parcerias", foi considerado uma primeira evidência dessa articulação. "Participação e protagonismo" foi a expressão apontada pelo grupo para descrever as distintas relações que podem envolver as articulações inter e intra-setoriais, seja em termos do envolvimento na ação de outros setores como colaborador, seja ocupando a posição de quem propõe e realiza a ação.

Quando se abordou o descritor 16, ficou claro que o envolvimento com as equipes de saúde da família, assim como com toda a rede básica, constituiria uma expressão estratégica da articulação acima referida. Da mesma maneira, a "participação social" (descritor 17), expressa pelo envolvimento dos movimentos sociais e instituições da sociedade civil na elaboração e realização das ações, refletiria uma articulação mais consolidada. Assim, esses dois descritores foram incorporados na redação final do descritor "articulação intra e intersetorial" (descritor final 7, tabela 1).

$\mathrm{O}$ "mapeamento dos serviços existentes" foi entendido como um item a ser abordado no diagnóstico situacional. Argumentou-se que o mapeamento, a atualização dessas listas e a sua divulgação seriam ações peculiares e de grande préstimo aos sistemas locais de saúde (descritor final 8, tabela 1). O descritor 14, "desenvolvimento de políticas a partir do diagnóstico", foi considerado inadequadamente formulado, dado que o NPAV não cria nem desenvolve políticas públicas, mas desenvolve um plano de ação, já então contemplado no descritor final 6. $\mathrm{O}$ "estímulo a pesquisas estratégicas" (descritor 18) foi reconhecido como adequado, mas o grupo sentiu necessidade de expressar exemplos a fim de demarcar as características e possibilidades dessa ação, evitando atribuições irreais ao escopo de abrangência dos NPAV (descritor final 9, tabela 1).

À "realização de ações contínuas de promoção à saúde" (descritor 19), reconhecida como um dos objetivos dos NPAV, foram acrescentadas ações de prevenção aos acidentes e violências. A "descrição das ações realizadas" (descritor 24) e a "capacidade de registro das ações" (descritor 30) foram consideradas indissociáveis da própria realização das atividades (binômio realização e registro das ações). A "capacidade de definir estratégias transversais abrangentes" (descritor 28) foi aí incluída, mudando-se o termo "definir" para "incorporar", no sentido de reconhecer que existem, já em curso, diversas ações dos vários programas de saúde onde a questão da prevenção da violência pode ser abordada (descritor final 10, tabela 1).

A "análise sistemática de dados encaminhada para gestor com propósito de intervenção" (descritor 23) foi reconhecida pelo grupo como importante. Entretanto, apontou-se que tais informações deveriam também subsidiar e fortalecer a ação dos parceiros dos demais setores, externos à saúde; assim, esse descritor foi incorporado ao de número 29 (descritor final 11, tabela 1).

Esta etapa de debates foi concluída com a reelaboração da lista inicial, permitindo um consenso em torno de 11 descritores finais listados na tabela 1. A partir desse conjunto de 11 descritores buscou-se, em seguida, identificar aqueles considerados os mais essenciais pelo grupo. Dessa nova lista gerada, cada membro escolheu individualmente, e sem interferências, cinco 
descritores que julgava os mais importantes (sem atribuir ordem de prioridade).

Os descritores mais votados pelo grupo foram (segundo a mesma numeração que aparece na tabela 1):

- descritor 1: monitoramento dos dados de mortalidade de acidentes e violência no município;

- descritor 2: apoio político, financeiro e administrativo (autonomia):

- descritor 3: construção do diagnóstico situacional adequado ao trabalho e à identidade do núcleo, incluindo o mapeamento dos serviços existentes;

- descritor 4: formação de equipe de trabalho (composição, dedicação e capacitação técnica); e

- descritor 7: articulação intra e intersetorial.

A lista dos cinco descritores foi novamente submetida ao escrutínio dos participantes, que atribuíram uma ordem de prioridade e uma nota a cada descritor. Empataram na primeira posição os itens 3 e 7 . Em seguida, na ordem de prioridade, surgiu o descritor 1, e na terceira posição, empatados, os descritores 2 e 4 . Uma vez analisadas as médias atribuídas, visando ao desempate de posições, a ordem final de relevância obtida foi:

- $1^{\mathrm{o}}$ : descritor 3. Construção do diagnóstico situacional.

- $2^{\circ}$ : descritor 7. Articulação intra e intersetorial.

- $3^{\circ}$ : descritor 1. Monitoramento dos dados de mortalidade de acidentes e violência no município.

- 40: descritor 2. Apoio político, financeiro e administrativo (autonomia).

- 5o: descritor 4. Formação de equipe de trabalho (composição, dedicação e capacitação técnica).

\section{DISCUSSÃO E CONCLUSÃO}

Os resultados apontam para a criação de 11 descritores, todos úteis para o desenvolvimento de indicadores de avaliação da implantação dos
NPAV. Indicam ainda a eleição, segundo a ótica dos atores, de cinco descritores considerados mais relevantes, sugerindo a atribuição futura de escores diferenciados destes entre si (respeitando-se sua hierarquia de prioridade) e em relação aos demais seis descritores. O desdobramento de elaboração de indicadores e de sua aplicação encontra-se atualmente em curso a partir de projeto financiado pelo Ministério da Saúde.

A análise dos cinco descritores priorizados e dos argumentos apresentados pelos coordenadores dos NPAV permite também inferir considerações que enriquecem a compreensão dessa trajetória, permitindo resgatar o contexto sócio-histórico das ações de enfrentamento da violência na saúde.

$\mathrm{O}$ primeiro aspecto que aparece de forma evidente é a questão relacionada à identidade de atuação, ainda em construção. Embora exista uma linha mestra de atuação preconizada pela portaria que institui os NPAV (10), o perfil das capacidades e competências já existentes em cada equipe acaba por orientar uma vertente de trabalho. Espera-se, entretanto, que essas opções sejam, de algum modo, coerentes com as situações apontadas no perfil de morbimortalidade dos municípios.

A clareza acerca da necessidade de um diagnóstico situacional para orientar o planejamento de cada núcleo também reflete a maturidade dos atores e da proposta de gestão dos NPAV. Todavia, para realizar tal empreitada, é essencial que os dados disponibilizados sejam confiáveis. Como tem sido apontado pela literatura (28), a qualidade dos registros de violências apresenta problemas antigos, que se revelam no percentual dos óbitos de causas externas mal definidas; revelam-se, também, na dificuldade de articulação entre os dados e os sistemas de informação das áreas de saúde e segurança pública, nas limitações do sistema de internações hospitalares, que não inclui os atendimentos de emergência, e na escassez de dados sobre violência doméstica, entre outros. As ações de enfrentamento das violências e acidentes realizadas pelo Ministério da
Saúde encontram-se majoritariamente vinculadas à Secretaria de Vigilância em Saúde (via Coordenação Geral de Vigilância de Agravos e Doenças Não Transmissíveis), e isso parece apontar para um cenário de investimento sólido para a superação de tais dificuldades, dada a reconhecida vocação dessa Secretaria para a qualificação de dados e informações.

A atuação intra e intersetorial constitui, sem dúvida, o grande desafio. Essa estratégia é apontada como condição fundamental para uma abordagem mais efetiva para o enfrentamento da violência $(3,8)$. A atuação em rede tem sido reconhecida como estratégia por excelência para a prevenção da violência, o cuidado de suas vítimas e familiares e a responsabilização dos autores de agressões $(5,29,30)$.

Assinalar a relevância do apoio institucional, financeiro e administrativo parece retratar a compreensão de que esse tripé é absolutamente indispensável à efetivação do trabalho. Estudos avaliativos para implantar a PNRMAV e outras ações dos governos municipais e estaduais têm revelado que raramente esses três aspectos do apoio institucional (político, financeiro e administrativo) caminham juntos, causando dificuldades à realização das ações propostas (31).

A escolha do descritor sobre a composição da equipe indica a preocupação com a precariedade de condições e recursos diante de missões institucionais de tal relevância. A sobrecarga e justaposição de funções e jornadas de trabalho não constituem cenários novos no setor saúde e ligamse sinergicamente à questão do apoio institucional (30). A capacitação das equipes para trabalhar com essa temática também é uma necessidade destacada em vários estudos para garantir a maior efetividade das ações (9).

Finalmente, a partir do acervo de descritores criados, deve-se ter em conta, para a subseqüente elaboração dos indicadores, que esses indicadores sejam claros, de fácil manejo, baseados em dados acessíveis e que empreguem estratégias metodológicas (de cunho quantitativo ou qualitativo) pertinentes. Os 11 descritores levantados neste 
estudo são igualmente úteis para esse propósito. Analisar os cinco mais votados leva ainda a questionar, do ponto de vista qualitativo, quais valores e qual contexto explicam o porquê da eleição destes descritores e não de outros. Revela um certo "quadro de relevância" dos operadores de uma área, nesse caso, a atuação no campo da violência em saúde.

\section{REFERÊNCIAS}

1. Minayo MCS. Violência: um problema para a saúde dos brasileiros. Em: Souza ER, Minayo MCS, orgs. Impacto da violência na saúde dos brasileiros. Brasília, DF: Ministério da Saúde; 2005. Pp. 9-41. Disponível em: bvsms. saude.gov.br/bvs/publicacoes/impacto violencia.pdf . Acessado em 19 de outubro de 2007.

2. Krug GE, Dahlberg LL, Mercy AJ, Zwi BA, Lozano R. Informe mundial sobre la violencia y la salud. Genebra: Organização Mundial da Saúde; 2003. Disponível em: http://www. paho.org/Spanish/AM/PUB/Contenido. pdf. Acessado em 19 de outubro de 2007.

3. Minayo MCS. The inclusion of violence in the health agenda: historical trajectory. Cienc Saude Coletiva. 2006;11(2):375-83.

4. Aguilar MJ, Ander-Egg E. Avaliação de serviços e programas sociais. Petrópolis: Vozes; 1994

5. Deslandes SF. Redes de proteção social e redes sociais: uma práxis integradora. Em: Lima CA, coord. Violência faz mal à saúde. Brasília, DF: Ministério da Saúde; 2004. Pp. 135-41.

6. Mello Jorge MHP, Gotlieb SLD. As condições de saúde no Brasil: retrospecto de 1979 a 1995. Rio de Janeiro: Fundação Instituto Oswaldo Cruz; 2000.

7. Reis AC, Ximenes LF, Souza ER, Minayo MCS. Morbidade hospitalar por causas violentas e a qualidade dos dados em 2001. Boletim CENEPI/CLAVES. 2003;10:1-10. Disponível em: http://www.bvsvs.cict. fiocruz. br/lildbi/docsonline/9/7/079-Boletim10. pdf. Acessado em 22 de outubro de 2007.

8. Brasil. Política nacional de redução de morbimortalidade por acidentes e violências. Brasília: Ministério da Saúde; 2001.

9. Minayo MCS, Deslandes SF. Análise diagnóstica da política nacional de saúde para redução de acidentes e violências. Rio de Janeiro: Editora Fiocruz; 2007.

10. Brasil. Portaria número 936-GM, em 19 de maio de 2004. Brasília: Ministério da Saúde; 2004. Disponível em: http://portal.saude. gov.br/portal/arquivos/pdf/portaria936. pdf. Acessado em 22 de outubro de 2007.

11. Pimenta Junior FG. Violência: prevenção e controle no Brasil [editorial]. Epidemiol Serv Saude. 2007;16(1):5-6.
12. Nirenberg O, Brawerman J, Ruiz V. El diseño de un modelo evaluativo. Em: Evaluar para la transformación: innovaciones en la evaluación de programas y proyectos sociales. Buenos Aires: Paidós; 2005. Pp. 133-65.

13. Raupp M, Reichele A. Avaliação: ferramenta para melhores projetos. Santa Cruz do Sul: EDUNISC; 2003.

14. Deponti CM, Ekert C, Azambuja JLB. Estratégia para construção de indicadores para avaliação da sustentabilidade e monitoramento de sistemas. Agroecol Desenvol Rur Sustent. 2002;3(4):44-52.

15. Assis S, Deslandes SF, Minayo MCS, Santos NC. Definição de objetivos e construção de indicadores visando a triangulação. Em: Minayo MCS, Assis SG, Souza ER, orgs. Avaliação por triangulação de métodos: abordagem de programas sociais. Rio de Janeiro: Fundação Instituto Oswaldo Cruz; 2005. Pp. 105-32.

16. Cohen E, Franco R. Avaliação de projetos sociais. Petrópolis: Vozes; 1994.

17. Furtado JP. Um método construtivista para avaliação em saúde. Cienc Saude Coletiva. 2001;6(1):165-81.

18. Mercado FJ, Bosi MLM. Avaliação não-tradicional de programas de saúde: anotações introdutórias. Em: Bosi MLM, Mercado FJ, orgs. Avaliação qualitativa de programas de saúde: enfoques emergentes. Petrópolis: Vozes; 2006. Pp. 11-21.

19. Akerman M, Mendes R, Bógus CM. Avaliação participativa em promoção da saúde: reflexões teórico-metodológicas. Em: Bosi MLM, Mercado FJ, orgs. Avaliação qualitativa de programas de saúde: enfoques emergentes. Petrópolis: Vozes; 2006. Pp. 145-60.

20. Wallerstein N. Powerlessness, empowerment and health: implications for health promotion programs. Am J Health Promot. 1992;6(3): 197-205.

21. Duggan EW, Thachenkary CS. Integrating nominal group technique and joint application development for improved systems requirements determinations. Inf Manag. 2004; 41(4):399-411.

22. Ivama AM, Rauber TL, Gomes SMT, Hofmeister MGS, Jaramillo NM. Prevenção e combate à falsificação e fraude de medicamentos: uma responsabilidade comparti-
Agradecimentos. Este estudo teve o apoio do Conselho Nacional de Desenvolvimento Científico e Tecnológico (CNPq) por intermédio de bolsas de produtividade e de iniciação científica. lhada. Brasília: Ministério da Saúde/Agência Nacional de Vigilância Sanitária/Organização Mundial da Saúde/Organização PanAmericana da Saúde; 2004.

23. Silva ACN, Bernardes RS, Moraes LRS, Reis JD. Critérios adotados para seleção de indicadores de contaminação ambiental relacionados aos resíduos sólidos de serviços de saúde: uma proposta de avaliação. Cad Saude Publica. 2002;18(5):1401-9.

24. Jones J, Hunter D. Consensus methods for medical and health services research. BMJ. 1995;311(7001):376-80.

25. Perry J, Linsley S. The use of the nominal group technique as an evaluative tool in the teaching and summative assessment of the inter-personal skills of student mental health nurses. Nurse Educ Today. 2006;26(4):346-53.

26. Potter M, Gordon S, Hamer P. The nominal group technique: a useful consensus methodology in physiotherapy research. N Z J Physiother. 2004;32(3):126-30.

27. Liakopoulos M. Análise argumentativa. Em: Bauer MW, Gaskell G, orgs. Pesquisa qualitativa com texto, imagem e som. Petrópolis: Vozes; 2002. Pp. 218-43.

28. Njaine K, Souza ER, Minayo MCS, Assis SG A produção da (des)informação sobre violência: análise de uma prática discriminatória. Cad Saude Publica. 1997;13(3):405-14.

29. Oliveira VLA, Pfeiffer L, Ribeiro CR, Gonçalves MT, Ruy IAE. Redes de proteção: novo paradigma de atuação - experiência de Curitiba. Em: Violência faz mal à saúde. Brasília, Distrito Federal: Ministério da Saúde; 2004. Pp. 143-52.

30. Njaine K, Assis SG, Gomes R, Minayo MCS. Networks for prevention of violence: from utopia to action. Cienc Saude Coletiva. 2006;11(2):429-38.

31. Gonçalves LAO, Sposito MP. Iniciativas públicas de redução da violência escolar no Brasil. Cad Pesqui. 2002;115:101-38.

Manuscrito recebido em 26 de julho de 2007. Aceito em versão revisada em 30 de novembro de 2007. 
ABSTRACT Objective. To explain the participatory model used to develop descriptors for evaluating the Accident and Violence Prevention Centers created by the Ministry of Health of Brazil to initiate and follow-up on public policies that reduce morbidity and

Participatory development of descriptors for evaluation of violence and accident prevention centers in Brazil mortality due to external causes.

Method. The nominal group technique was the main strategy employed. Participants included 13 representatives from centers in the states of Rio de Janeiro and Espírito Santo.

Results. Eleven descriptors were identified for evaluation, with five being selected as priorities: situation analysis; intra- and intersectoral processes; monitoring of municipal accident and mortality data; political, financial, and administrative support (autonomy); and establishment of a work team (composition, dedication, and technical skills).

Conclusions. The participatory model revealed both a step toward identity-developing for the centers, as well as the history of the health sector's actions in this area. The process also highlighted old barriers that must be overcome if effective actions against violence are to be taken by the country.

Key words External causes, violence/prevention \& control, Brazil. 